\title{
Growth and Etching of Centimeter-scale Self-assembly Graphene- $h$-BN Super-ordered Arrays: Implications for Integrated Electronic Devices
}

Menghan Li, ${ }^{1, \#}$ Yajing Sun, ${ }^{2, \#}$ Xiao Xue, ${ }^{3}$ Xueying Lu, ${ }^{1}$ Zilong Guo, ${ }^{1}$ Yandong Han, ${ }^{1}$ Jichen Dong, ${ }^{3}$ Dechao Geng, ${ }^{2}$ Lin Li, ${ }^{1,}{ }^{*}$ Wensheng Yang ${ }^{1}$

${ }^{1}$ Institute of Molecular Plus, Tianjin University, Tianjin 300072, China

2 Tianjin Key Laboratory of Molecular Optoelectronic Sciences, Department of Chemistry, School of Science, Tianjin University, Tianjin 300072, China

${ }^{3}$ Beijing National Laboratory for Molecular Sciences, Key Laboratory of Organic Solids, Institute of Chemistry, Beijing, 100190 P. R. China

Email: linli2020@tju.edu.cn

\# These authors contributed equally to this work. 


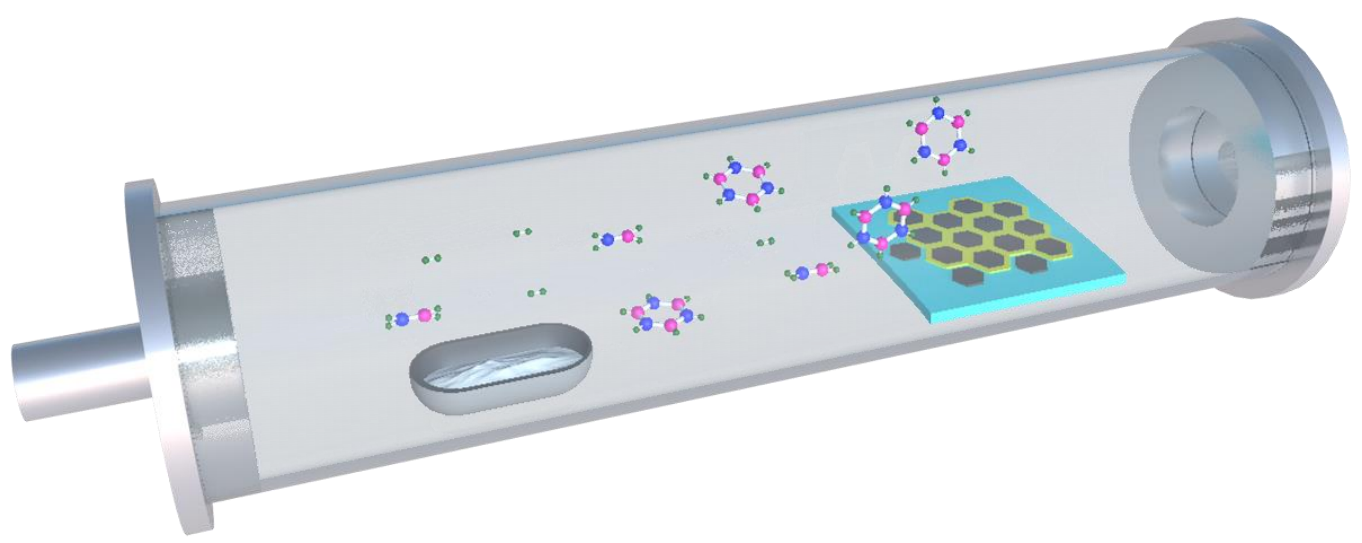

Figure S1. Schematic representation of the experimental tube-in-tube CVD setup for the growth of graphene- $h$-BN heterostructures. 


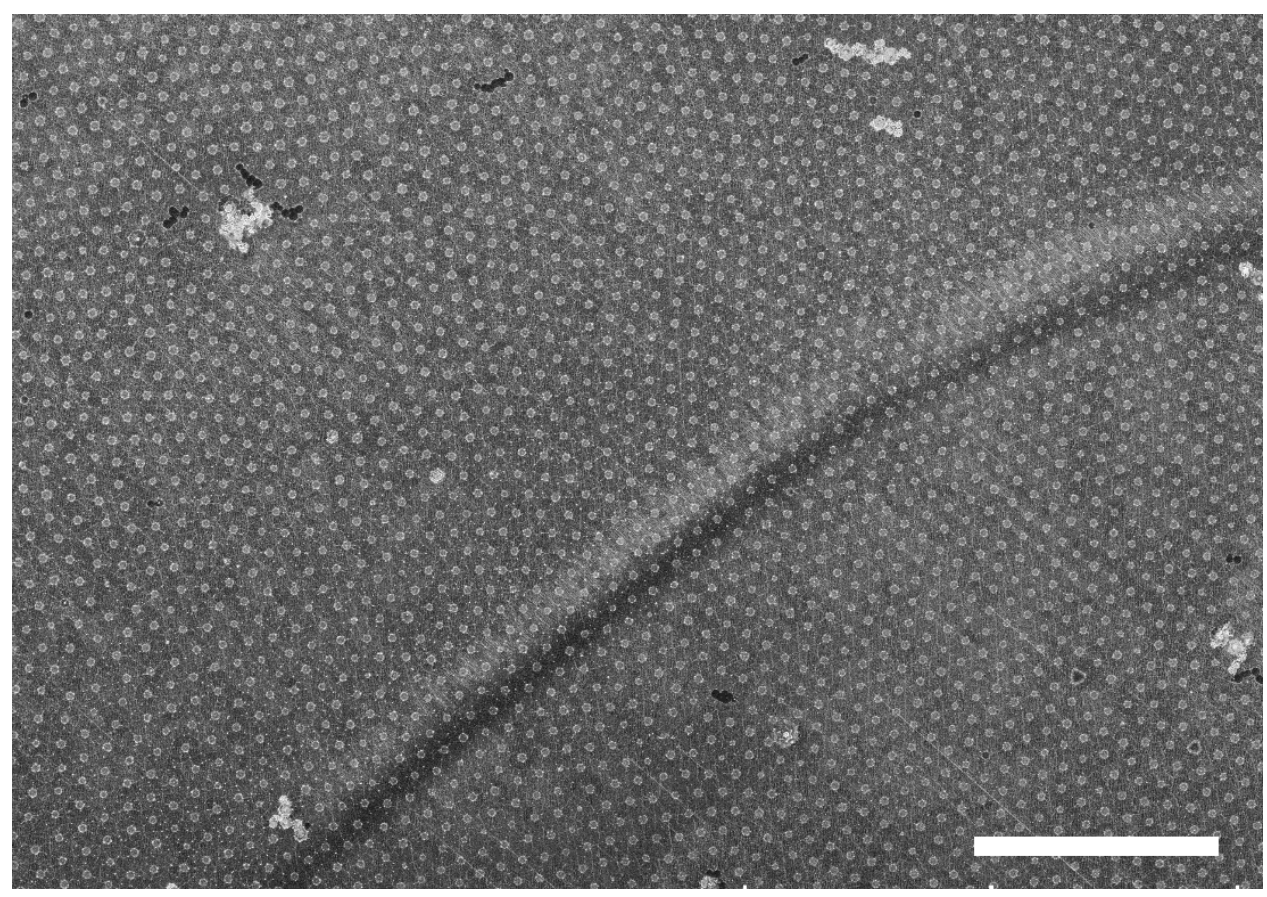

Figure S2. SEM image of the large area graphene- $h$-BN arrays on the $\mathrm{Cu}$ substrate. The scale bar is $50 \mu \mathrm{m}$. 

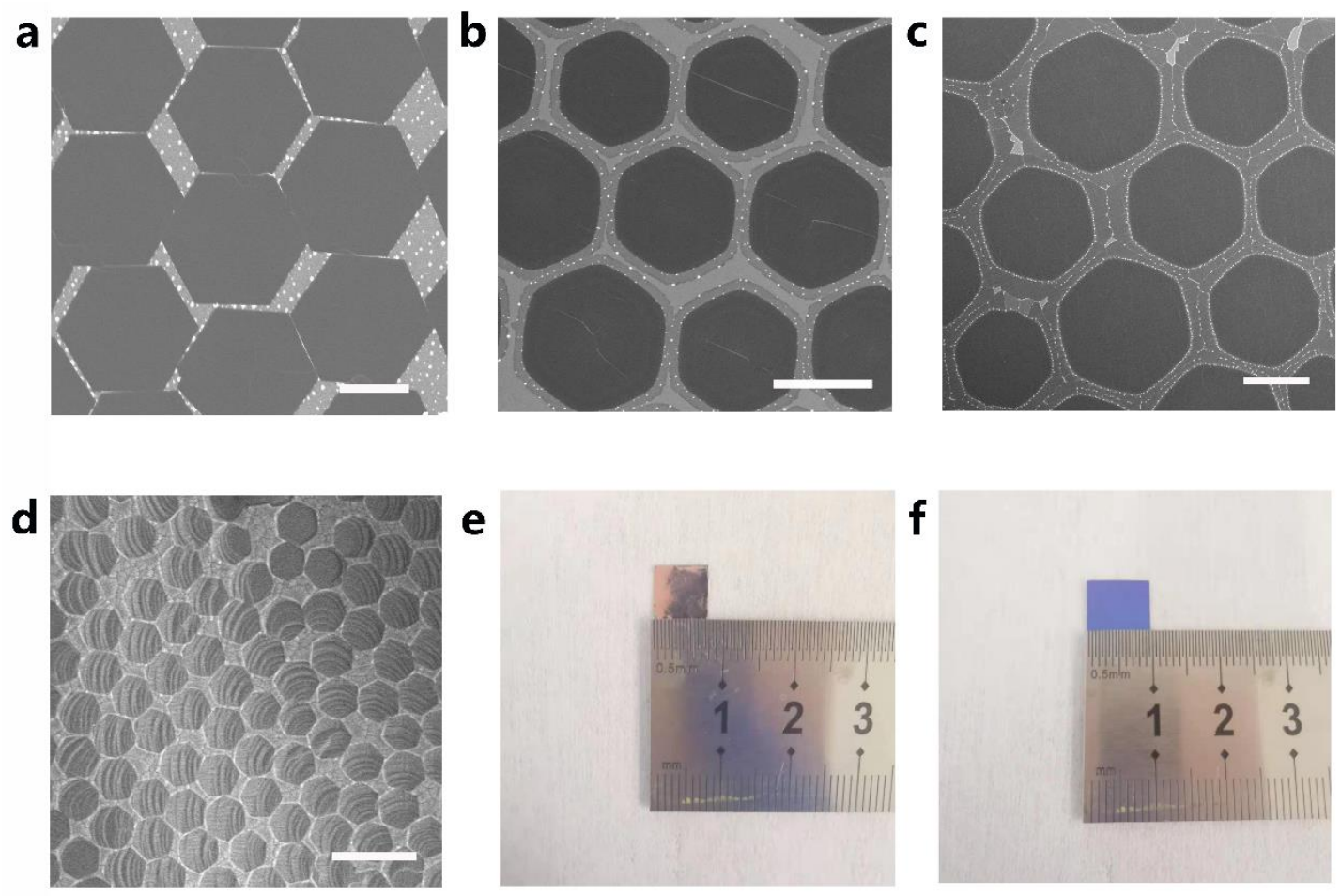

Figure S3. (a)-(c) SEM images of super-ordered arrays at different growth stages.

There is only graphene array in (a). $h$-BN grow outward along the graphene in (b) and, $h$-BN extends more that almost filling the entire gap in (c). (d) SEM image of graphene single crystal array. (e) shows a larger area of the array and (f) shows a film transferred onto $\mathrm{Si} / \mathrm{SiO}_{2}$ substrate, with an area of up to 1 square centimeter. The scale bars in (a)(c) are $5 \mu \mathrm{m}$ and in (d) is $20 \mu \mathrm{m}$. 
a
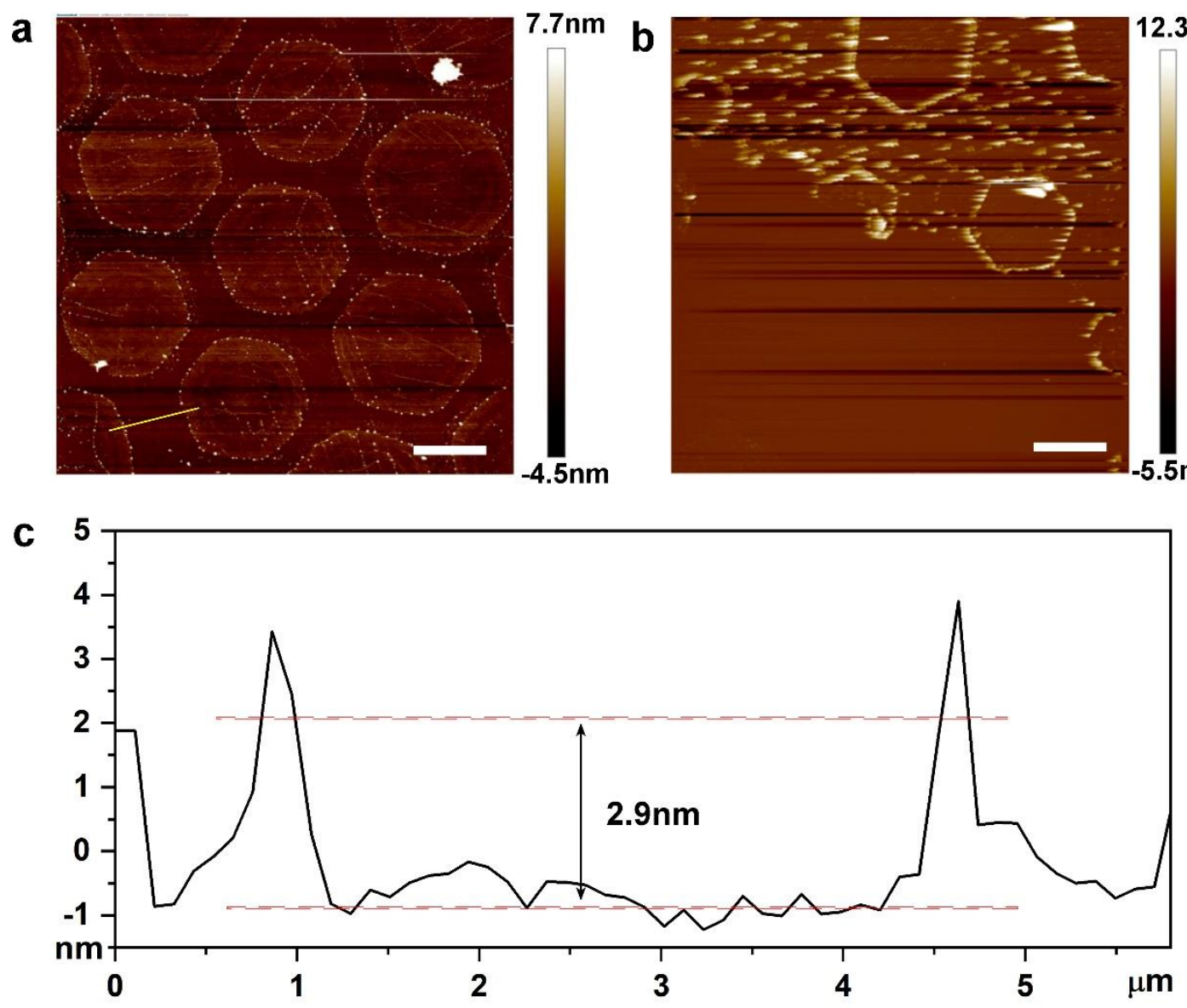

Figure S4. AFM images of single graphene- $h$-BN arrays. (a) A selected portion within the whole film. (b) AFM image of the film boundary. (c) Thickness profile of the single flake marked by yellow in (a), confirming the uniform surface of the graphene and $h$ BN monolayers. The scale bars in (a) and (b) are $2 \mu \mathrm{m}$. 


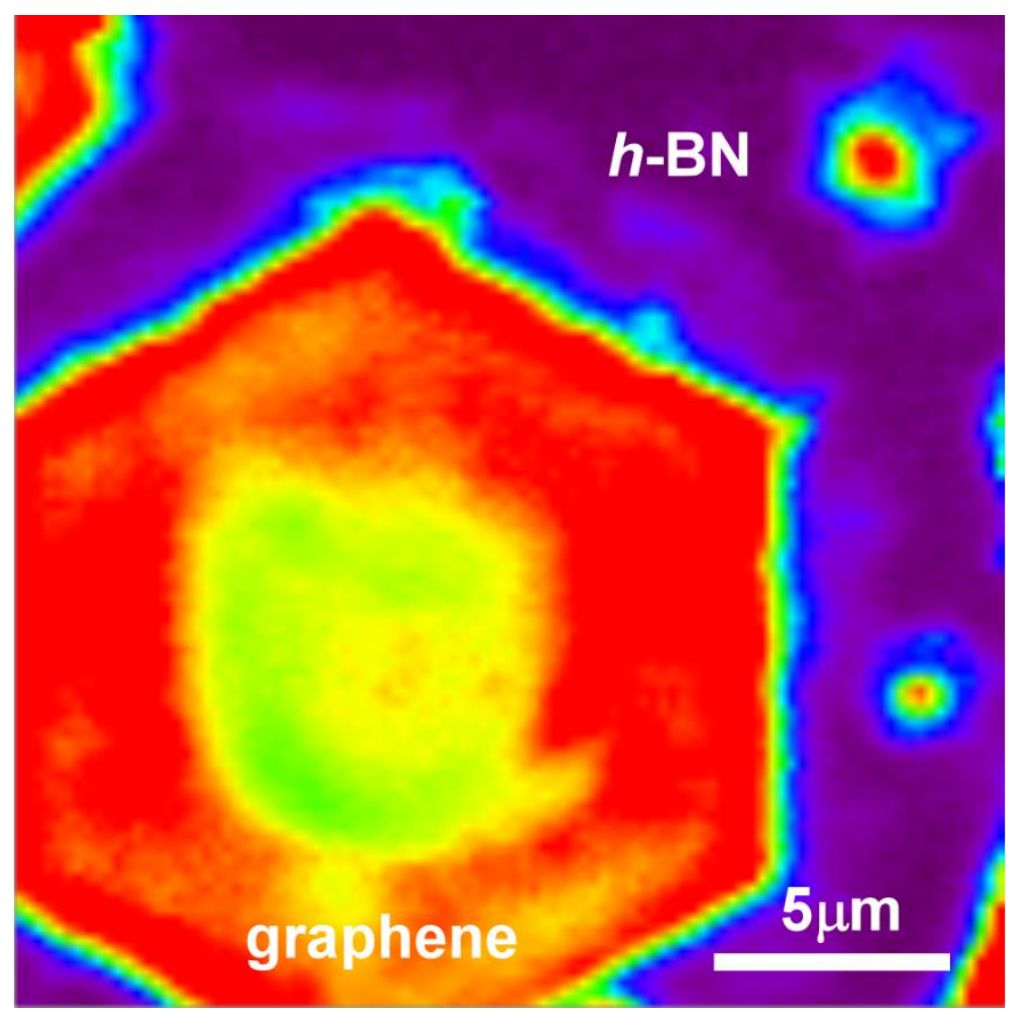

Figure S5. Raman mapping images of the graphene- $h$-BN film. 

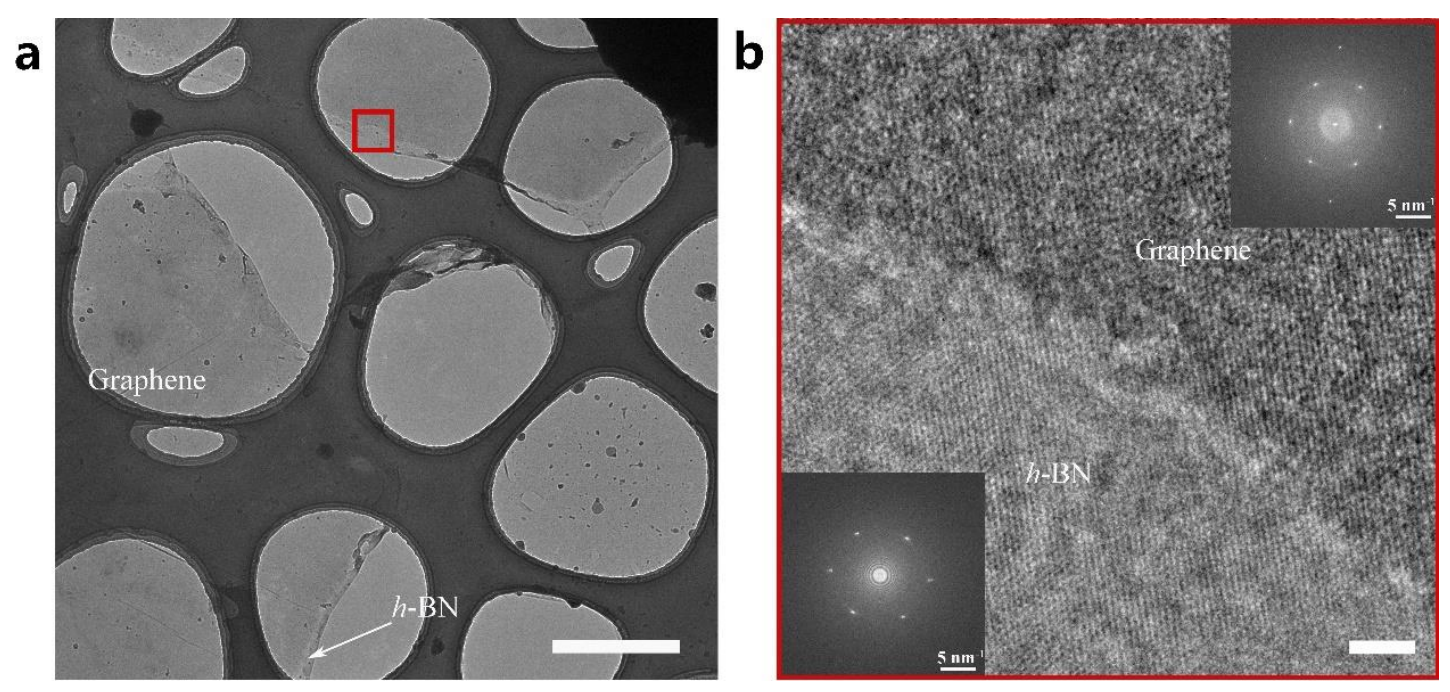

Figure S6. TEM images of the planar heterostructure. (a) Morphologies of heterostructure, with different sites marked. The edge shows that graphene and h-BN are atomic continuous (different contrast). (b) The high resolution of the red-framed part in (a). Insets: fast Fourier transform images of graphene (the top right corner) and h-BN (left bottom). The scale bars in (a) is $2 \mu \mathrm{m}$ and in (b) is $2 \mathrm{~nm}$. 


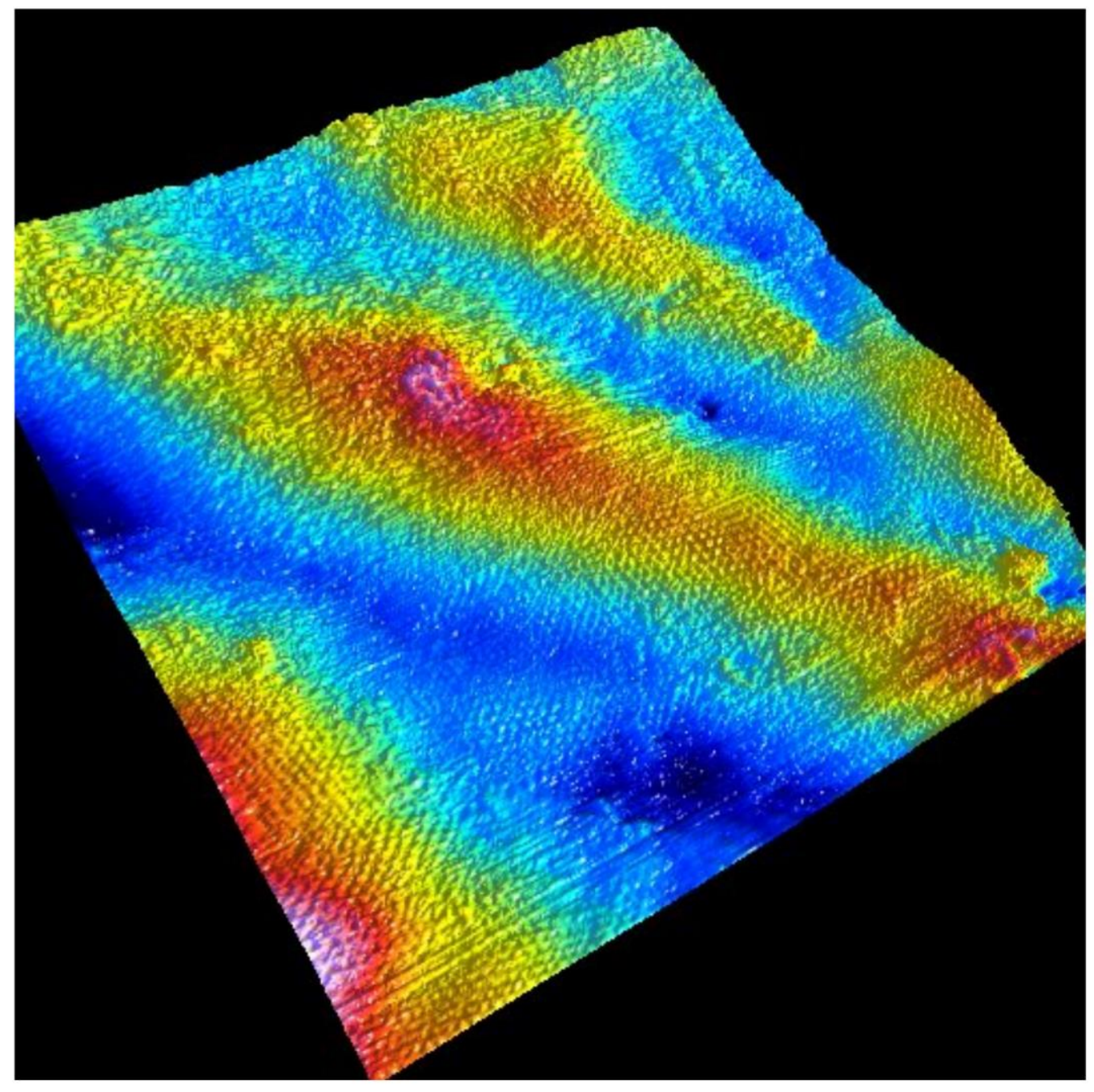

Figure S7. (a) 3D image of the boundary between graphene and $h$-BN. 

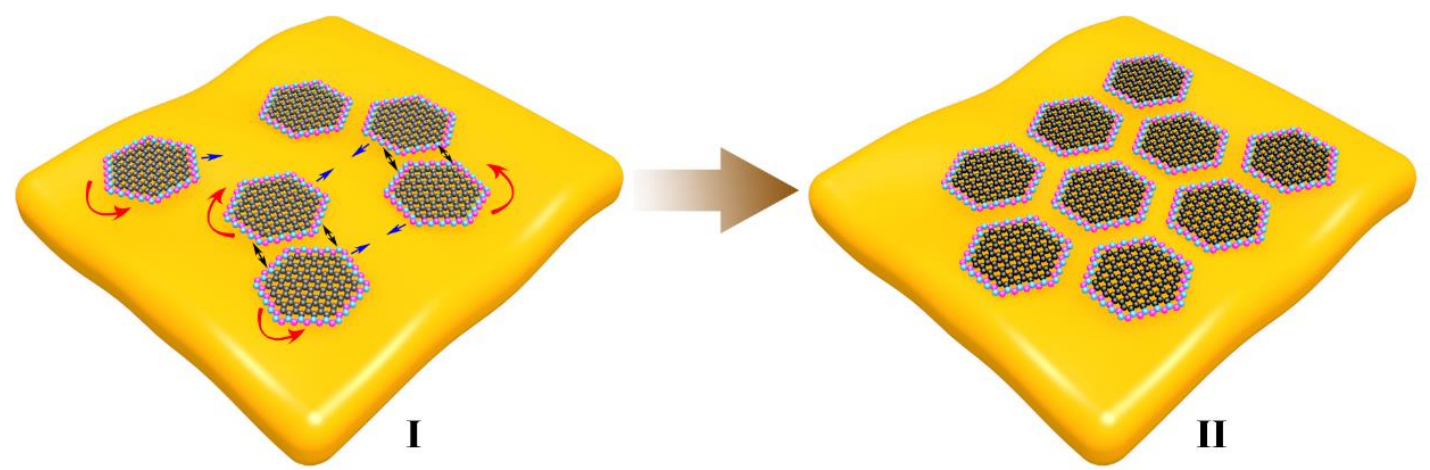

Figure S8. Schematic diagram of electrostatic interactions that induce the assembly of graphene- $h$-BN structure over a large area. The heterostructure units move and rotate on the liquid metal surface and spontaneously form regular array structures, owing to the action of electrostatic potential. 
a

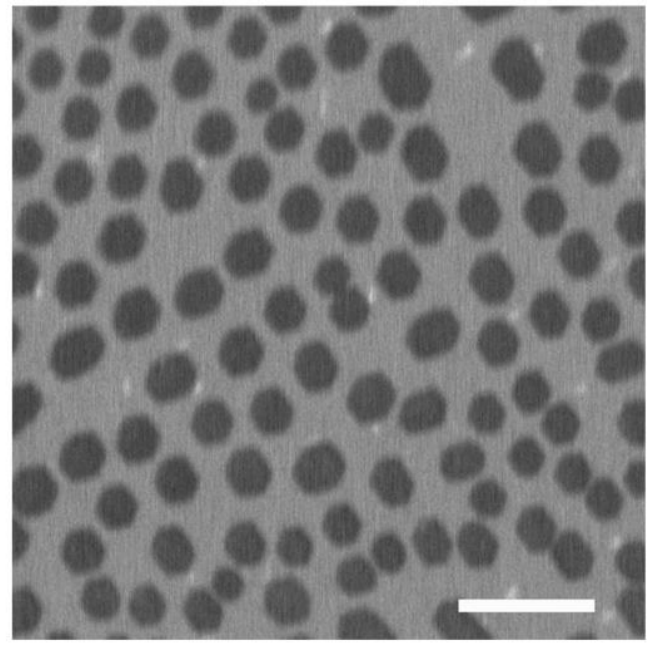

b

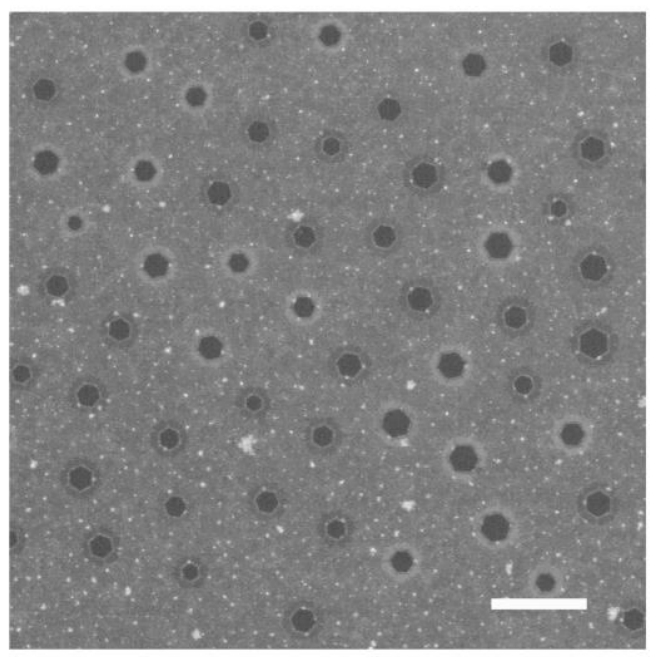

Figure S9. (a) SEM image of array of graphene single crystal with a size about $1 \mu \mathrm{m}$. (b) SEM images of graphene and heterostructures at intermediate growth stage. The scale bars are $5 \mu \mathrm{m}$. 

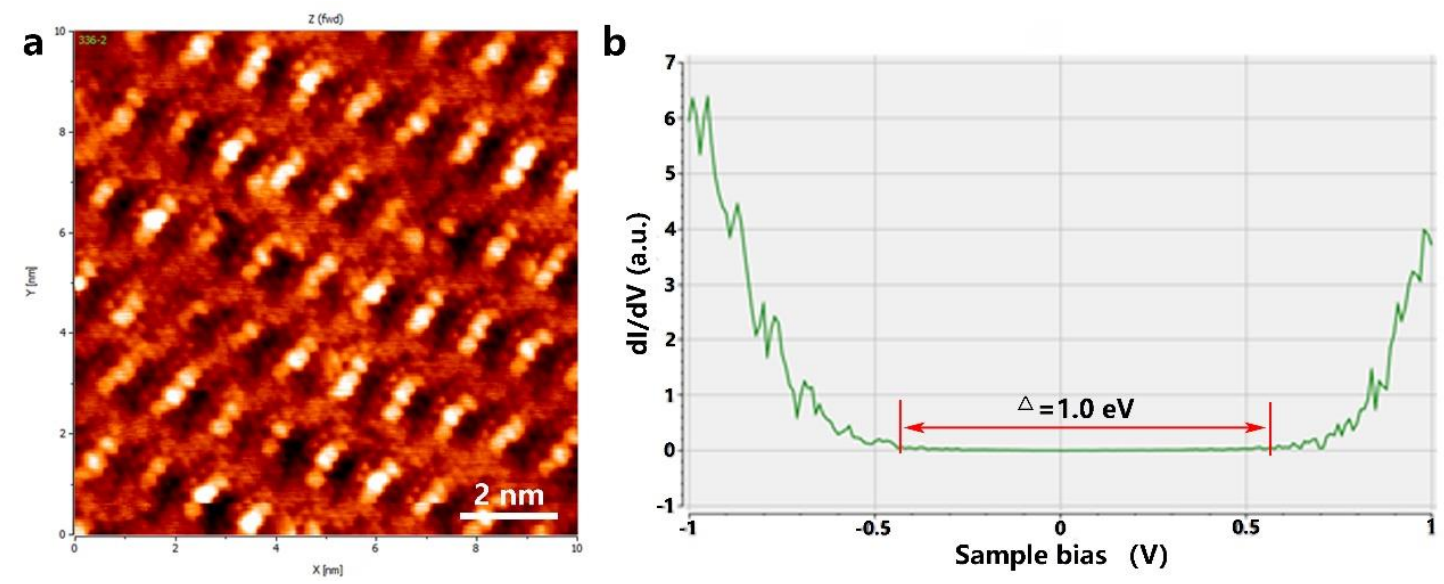

Figure S10. (a) Images of atoms corresponding to the graphene being measured. STM $\mathrm{dI} / \mathrm{dV}$ spectra taken near the heterogeneous boundary shown in (b) and the band gap is calculated to be about $1.0 \mathrm{eV}$. 
a

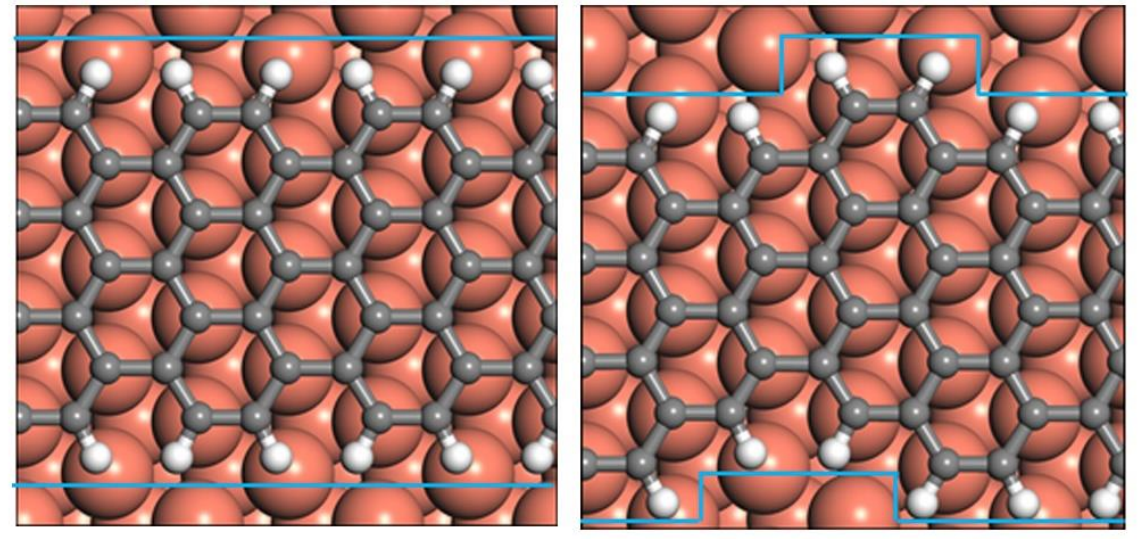

b
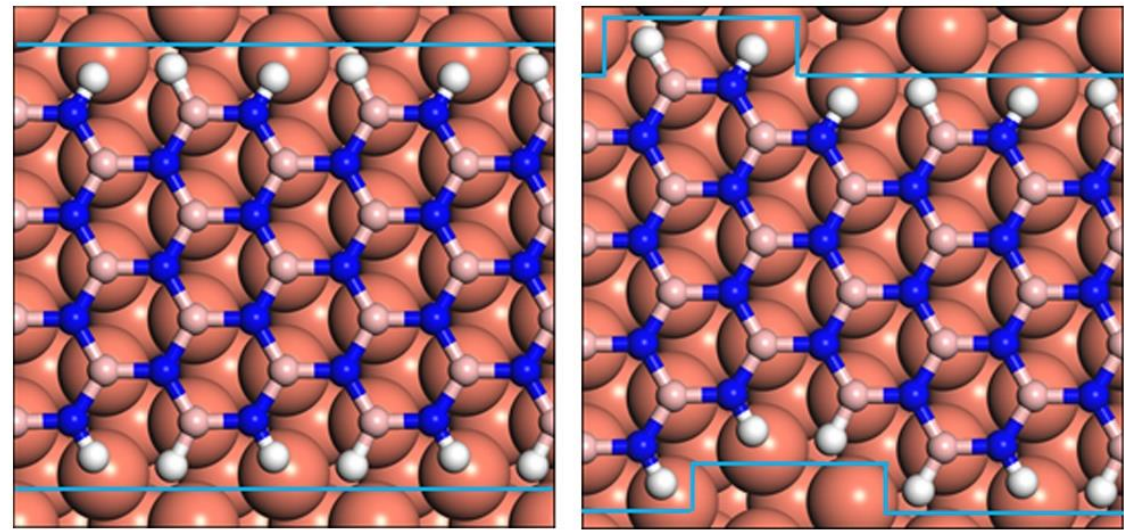

Figure S11. (a) Atomic simulation of graphene crystal on $\mathrm{Cu}$ (111) plane. (b) Atomic simulation of $h$-BN crystal (on copper (111) plane). 
Table S1. The conditions for the growth of graphene are shown in the picture.

\begin{tabular}{ccccc} 
Sample & $\mathbf{C H}_{4}(\mathbf{s c c m})$ & $\mathbf{H}_{2}(\mathbf{s c c m})$ & $\operatorname{Ar}(\mathbf{s c c m})$ & Growing time(min) \\
\hline I & 1.5 & 100 & 100 & 5 \\
II & 1.8 & 100 & 100 & 5 \\
III & 2.0 & 100 & 100 & 5 \\
\hline
\end{tabular}

